\title{
Redes Sociais e Fabricação Digital na Construção de Objetos para Apoio a Atividades Educacionais
}

\author{
André Peres ${ }^{1}$, Evandro Manara Miletto ${ }^{1}$, Fabiana Lorenzi ${ }^{2}$, Elidiane Zayaeskoski ${ }^{1}$, \\ Ramon Costa da Silva ${ }^{1}$, Gianfranco Meneguz ${ }^{1}$ \\ ${ }^{1}$ Instituto Federal de Educação, Ciência e Tecnologia \\ do Rio Grande do Sul (IFRS) Campus Porto Alegre \\ Rua Cel Vicente, 281 - Porto Alegre, RS - Brasil \\ \{andre.peres, evandro.miletto\}@poa.ifrs.edu.br \\ \{ezayaeskoski;gmeneguz; ramondasilv\}@gmail.com \\ ${ }^{2}$ Universidade Luterana do Brasil (ULBRA) Campus Canoas \\ Av. Farroupilha, 8001 - Canoas, RS - Brasil \\ fabilorenzi@gmail.com
}

\begin{abstract}
This paper describes an technological structure made to the proposition, fabrication and publication of physical objects to be used in educational activities. The proposition, definition and specification of the objects are made in a collaborative way through a specific virtual social network and the fabrication is made in a digital fabrication lab. After the fabrication and tests, the created object is published over the Internet in a public repository, allowing the object to be recreated, studied, utilized and modified by the community.
\end{abstract}

Resumo. Este artigo descreve uma estrutura tecnológica criada para a proposição, fabricação e divulgação de objetos físicos para apoio a atividades educacionais. A proposição, definição e especificação dos objetos é feita de forma colaborativa através de uma rede social virtual específica e a fabricação realizada utilizando-se um laboratório de fabricação digital. Após a fabricação e testes, o objeto criado é divulgado na Internet em um repositório público para que seja recriado, estudado, utilizado e modificado pela comunidade.

\section{Introdução}

As novas tecnologias computacionais aliam-se às novas demandas da sociedade por produtos e serviços cada vez mais pessoais, interativos e acessíveis. A popularização e redução de custos dos equipamentos eletrônicos permitem que novas soluções sejam criadas fora dos grandes centros de desenvolvimento. Esta possibilidade dá espaço para a criação de uma nova geração de pessoas motivadas pela cultura do "faça você mesmo"(do it yourself) também conhecida como geração "maker".

O crescimento da comunidade maker resultou na criação de espaços de colaboração denominados makerspaces (espaços para criação de qualquer tipo de objeto, tecnológico ou não); hackerspaces (com foco mais voltado para tecnologia); e fablabs (laboratórios com objetivos e equipamentos comuns, conectados em uma rede mundial de labs) [Makezine 2015]. 


\section{CBIE-LACLO 2015}

Anais dos Workshops do IV Congresso Brasileiro de Informática na Educação (CBIE 2015)

Estes espaços ganham importância fundamental pois, apesar da diminuição constante dos custos de aquisição destes equipamentos, é de grande valia a sua disponibilização em um ambiente de criação colaborativo para que seus usuários (os makers) possam trocar conhecimento, capacitarem-se e construírem juntos novas soluções.

Este artigo apresenta a descrição da criação de uma infraestrutura tecnológica capaz de produzir uma coleção de objetos educacionais em um laboratório baseado no modelo da fablab. Esta coleção é composta por objetos físicos providos de sensores, atuadores e interface de comunicação em rede para uso em experimentos educacionais.

A partir desta estrutura, pode-se utilizar os objetos em experimentos em sala de aula, permitindo que se estabeleça uma relação mais próxima entre conceitos teóricos explorados em aula e a prática, através da fabricação, uso e modificação de objetos criados a partir da fabricação digital.

Os objetos desenvolvidos são publicados em um repositório na internet, permitindo que sejam criados, utilizados e modificados em outros espaços de criação colaborativos (makerspaces, hackerspaces e fab labs) por estudantes e professores em atividades de ensino.

\section{As Novas Tecnologias e a Educação}

A utilização de espaços de criação (e o próprio conceito de aprender fazendo) está alinhado com as teorias construtivistas de Piaget. Para Piaget, o "... uso de métodos ativos que dão vasto campo de pesquisa espontânea para a criança ou adolescente e exige que cada nova verdade a ser aprendida, ser redescoberta, ou pelo menos reconstruída pelo estudante e não simplesmente importada para ele.". Além disso, ainda para Piaget, professores dos níveis secundário e universidade deveriam, além de conhecer os conteúdos a serem ministrados, realizar uma abordagem com ponto de vista interdisciplinar [[Martinez and Stager 2013] apud [Piaget 1976]].

Em sintonia com a abordagem interdisciplinar de Piaget, o pesquisador Seymour Papert considera que: "na nossa imagem de laboratório de computação em uma escola, um papel importante é desempenhado por inúmeras 'portas de controladores', que permitem que qualquer estudante conecte qualquer dispositivo no computador ... o laboratório terá um conjunto de motores, solenoides, relés, sensores, etc. .. Ao usá-los, os alunos serão capazes de inventar e construir uma infinita variedade de sistemas cibernéticos." [[Martinez and Stager 2013] apud [Papert and Solomon 1971]].

O uso de eletrônicos de baixo custo, sensores e controladores começou com Papert, Mitchel Resnick e Fred Martin na década de 1990 usando o kit Lego Mindstorms [Blikstein 2013]. Pode-se citar também a construção de projetos de robótica, como no trabalho intitulado "Utilização de Hardware Livre Arduino em Ambientes de Ensinoaprendizagem" [Alves et al. 2012]. Em [Alves et al. 2012] destaca-se a qualificação do uso da robótica no ensino e aprendizagem no ponto de vista construtivista de Piaget, Vygotsky e Papert.

A partir destes experimentos iniciais, sempre que novas tecnologias emergem, são criados estudos sobre como usá-las em atividades de aprendizagem. O trabalho de [Farias et al. 2014], por exemplo, utiliza a fabricação de soluções tecnológicas utilizando controladores através de atividade de extensão, como um meio de melhorar o 
CBIE-LACLO 2015

Anais dos Workshops do IV Congresso Brasileiro de Informática na Educação (CBIE 2015)

desempenho de alunos ingressantes em cursos de computação.

\section{Fabricação Digital Colaborativa de Objetos Reais de Aprendizagem}

A infraestrutura implementada visa permitir que um grupo de pessoas possa propor, especificar e desenvolver objetos de aprendizagem através de um ambiente colaborativo virtual e, posteriormente à definição do objeto, fabricá-lo utilizando equipamentos de fabricação digital.

De acordo com a rede fablab, este espaço agrupa um conjunto de máquinas por comando numérico, tais como: impressoras $3 \mathrm{D}$, máquinas de corte a laser, fresadoras de precisão e de grande porte e plotters de recorte [Eychenne and Neves 2013].

Após a definição e fabricação, é realizada a publicação do projeto do objeto, tornando possível que qualquer pessoa com acesso a estes recursos tecnológicos seja capaz de estudar e reconstruir os objetos de aprendizagem desenvolvidos.

A construção dos conceitos educacionais a serem trabalhados e desenvolvimento do projeto se dá através da colaboração. Para que seja criado o ambiente de colaboração e também para que se tenha o registro desta atividade, foi disponibilizada um sistema que implementa uma rede social virtual (RSV) do laboratório de fabricação digital. O processo de criação de um objeto é apresentado na figura 1.

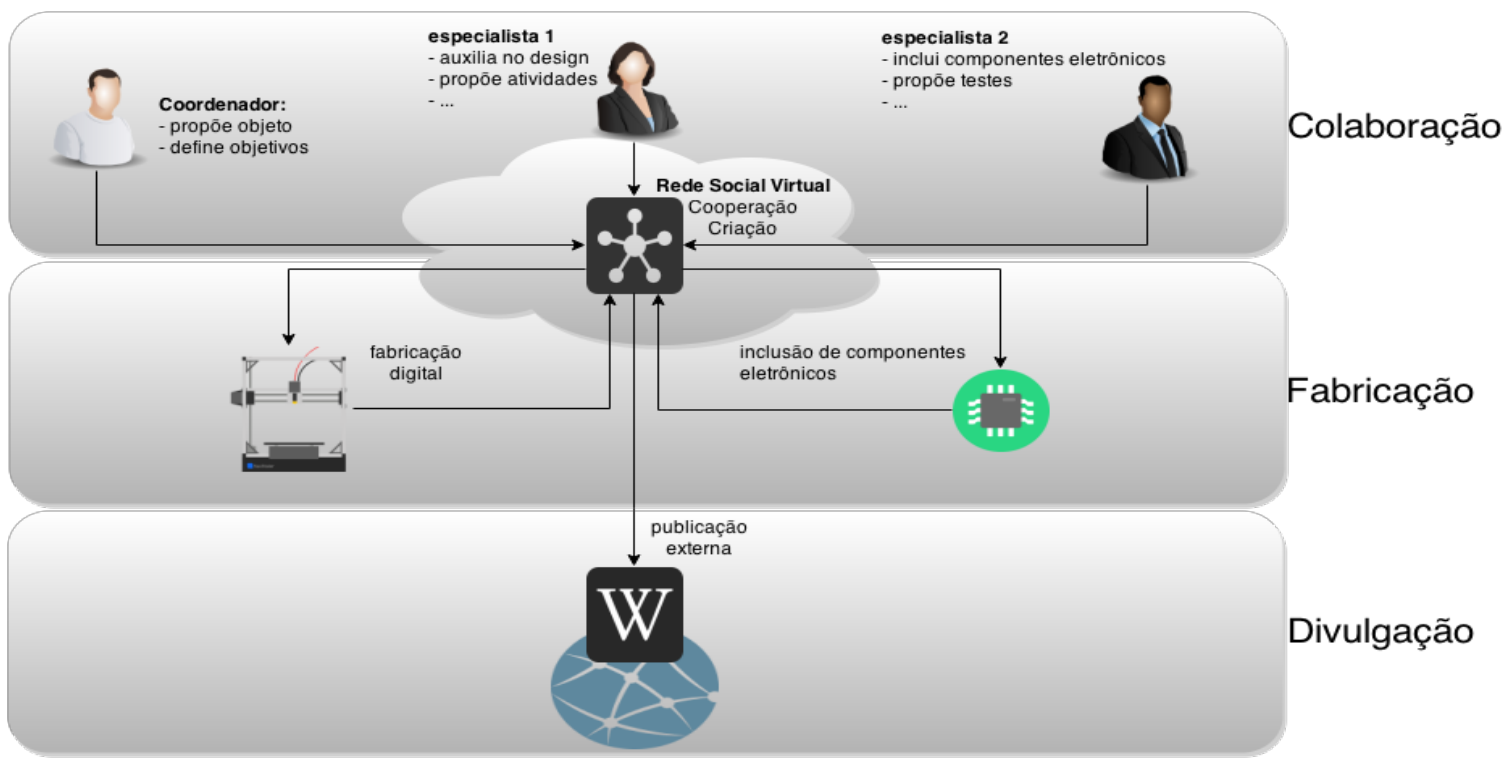

Figura 1. Processo de Criação e Publicação de um Objeto

Inicialmente um usuário da rede social propõe um objeto e define seus objetivos. Usuários especialistas contribuem de forma colaborativa na descrição deste objeto que posteriormente é prototipado. Ao final do processo o objeto é publicado.

O sistema utilizado para implementação da rede social é o humhub [HumnHub 2015]. Este sistema possui uma interface familiar aos usuários de redes sociais e permite a criação de espaços de discussão entre grupos de usuários. Os usuários envolvidos no projeto cadastram-se no sistema e, para cada objeto de aprendizagem a ser elaborado, é criado um espaço de discussão. 


\section{CBIE-LACLO 2015}

Anais dos Workshops do IV Congresso Brasileiro de Informática na Educação (CBIE 2015)

No espaço de discussão de um objeto são apresentados os objetivos do objeto e é iniciada a sua elaboração teórica. A figura 2 apresenta a página da rede social e o espaço de criação de uma turbina eólica a ser utilizada em sala de aula em experimento de geração de energia.

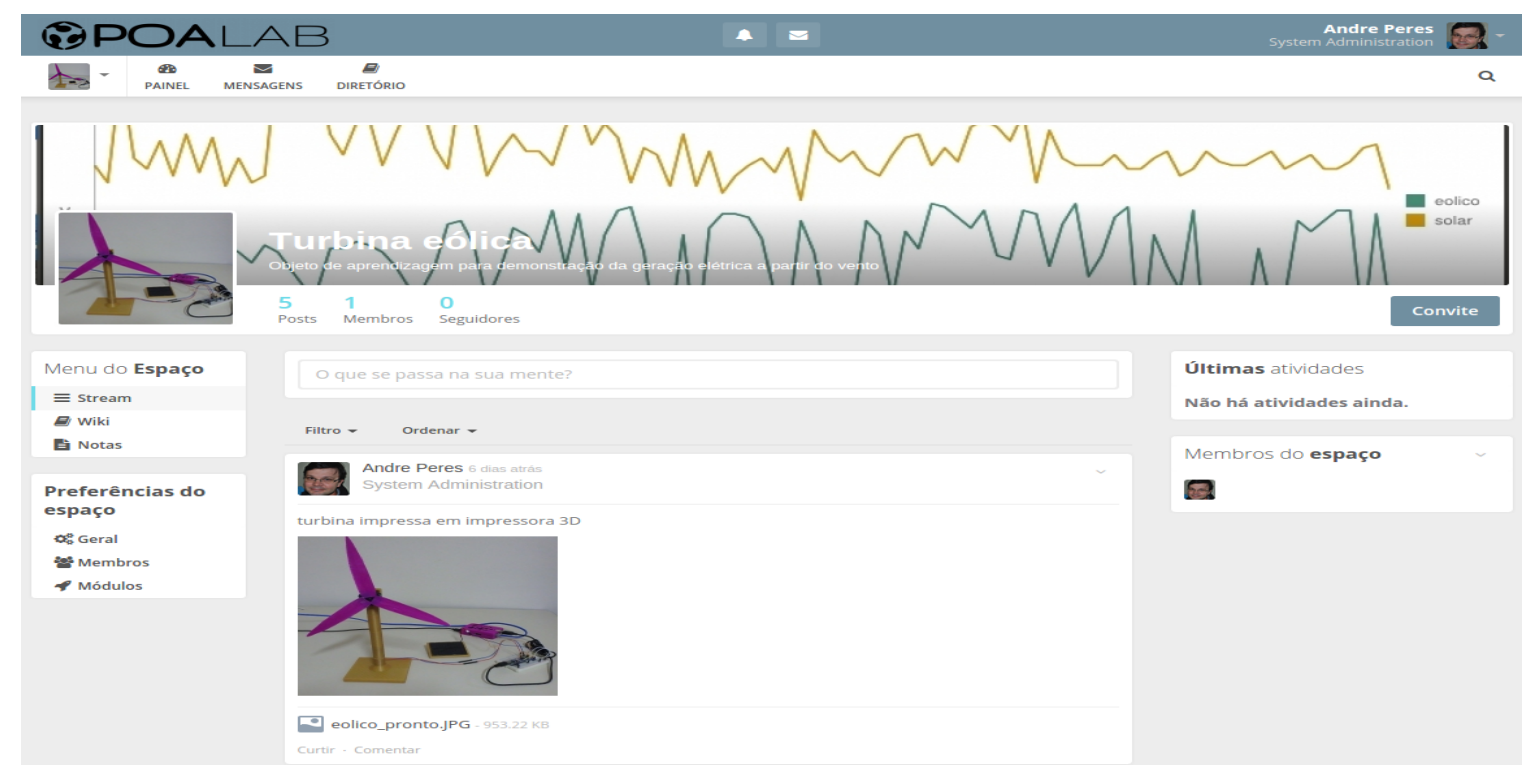

Figura 2. Página da Rede Social do Laboratório de Fabricação Digital

O proponente do objeto encarrega-se da coordenação do espaço, tendo como responsabilidade permitir a colaboração entre os envolvidos e orientar os trabalhos. Isto evita os problemas no uso de redes sociais em atividades de ensino, tais como falta de regras explícitas, pouca discussão e problemas de hierarquia apontados por [Mota et al. 2014].

Ao final do processo de definição inicial, o objeto passa a ser fabricado no laboratório. Neste momento a colaboração passa a ser presencial e todo o trabalho deve ser documentado no espaço criado na rede social. O laboratório em uso atualmente é apresentado na figura 3 .



Figura 3. Laboratório de Fabricação Digital 


\section{CBIE-LACLO 2015}

Anais dos Workshops do IV Congresso Brasileiro de Informática na Educação (CBIE 2015)

Este laboratório possui um conjunto de equipamentos controlados por comando numérico (CNC ou Computerized Numeric Control Machines), ou seja, são operados a partir de arquivos de computador. A disponibilização de equipamentos CNC permite que um usuário do espaço possa, a partir de um programa de desenho vetorial 2D ou de renderização em 3D, construir objetos sólidos em polímeros (ABS, PLA, acrílico, etc), metal ou madeira e, se desejar, equipar este objeto com componentes eletrônicos. Os equipamentos disponíveis permitem:

- impressoras 3D - construção de objetos plásticos (ABS e PLA) com dimensões de até $20 \mathrm{~cm} \times 20 \mathrm{~cm}$ x $20 \mathrm{~cm}$ a partir de arquivos $3 \mathrm{D}$;

- plotter de recorte - corte de adesivos de vinil, papel, EVA, etc, com até $60 \mathrm{~cm}$ de largura a partir de arquivos vetoriais 2D;

- máquina de corte a laser - recorte de madeira, $\mathrm{MDF}$, acrílico, etc. de até $40 \mathrm{~cm} \mathrm{x}$ $60 \mathrm{~cm}$ a partir de um arquivo vetorial 2D;

- fresadora de precisão - fresa (ferramenta rotativa de usinagem) de madeira, MDF, acrílico, placas de cobre para confecção de placas de circuitos eletrônicos, etc, com até $20 \mathrm{~cm} \times 25 \mathrm{~cm} \times 15 \mathrm{~cm}$ a partir de arquivos 2D e 3D (não visível na figura 3); extrusora para plástico - equipamento com capacidade de fabricar filamento para impressora 3D a partir de pedaços de plástico provenientes de descarte (não visível na figura 3); estação de solda - para soldagem de componentes eletrônicos.

Após a fabricação, todos os arquivos necessários para a construção do objeto final são adicionados ao espaço do objeto que agora passa a ser utilizado para questionamentos, registros de casos de uso e modificações.

Quando um objeto apresenta um histórico positivo de uso dentro da comunidade de usuários da rede social do laboratório, é publicado em um repositório externo do tipo wiki com licença Creative Commons Atribuição 4.0 Internacional, permitindo seu compartilhamento, adaptação e uso.

Experiências utilizando páginas Wiki de forma colaborativa são fonte de pesquisas atuais, como em [Oliveira and Ferreira 2014]. Neste trabalho os autores utilizam a wiki para criação textual colaborativa entre alunos. Aproveitando-se da forma como a wiki pode ser utilizada, decidiu-se que esta é a melhor maneira de descrever os objetos, as instruções, alguns exemplos de experiências e, ao mesmo tempo, fornecer todos os arquivos necessários para sua reconstrução. Para tanto, construiu-se um servidor Web com o software MediaWiki [MediaWiki 2014] para servir como repositório de objetos.

A wiki serve não apenas como repositório, mas também como forma de interação com usuários externos ao laboratório. Qualquer pessoa pode usar a página wiki e criar novas entradas para um objeto diferente.

A Figura 4 mostra a tela da wiki criada com a entrada descrevendo como construir uma turbina eólica, circuito a ser criado, passo-a-passo e arquivos necessários para a construção deste objeto.

Definiu-se que para que um objeto seja publicado na wiki é necessário que tenha: a descrição do objeto e seus objetivos; a listagem dos componentes eletrônicos necessários; a descrição esquemática do circuito; imagens, fotos, detalhes importantes; arquivos a 
CBIE-LACLO 2015

Anais dos Workshops do IV Congresso Brasileiro de Informática na Educação (CBIE 2015)

serem utilizados para fabricação digital; código fonte necessário (controlador e interface com computador); e exemplos de uso em atividades educacionais.

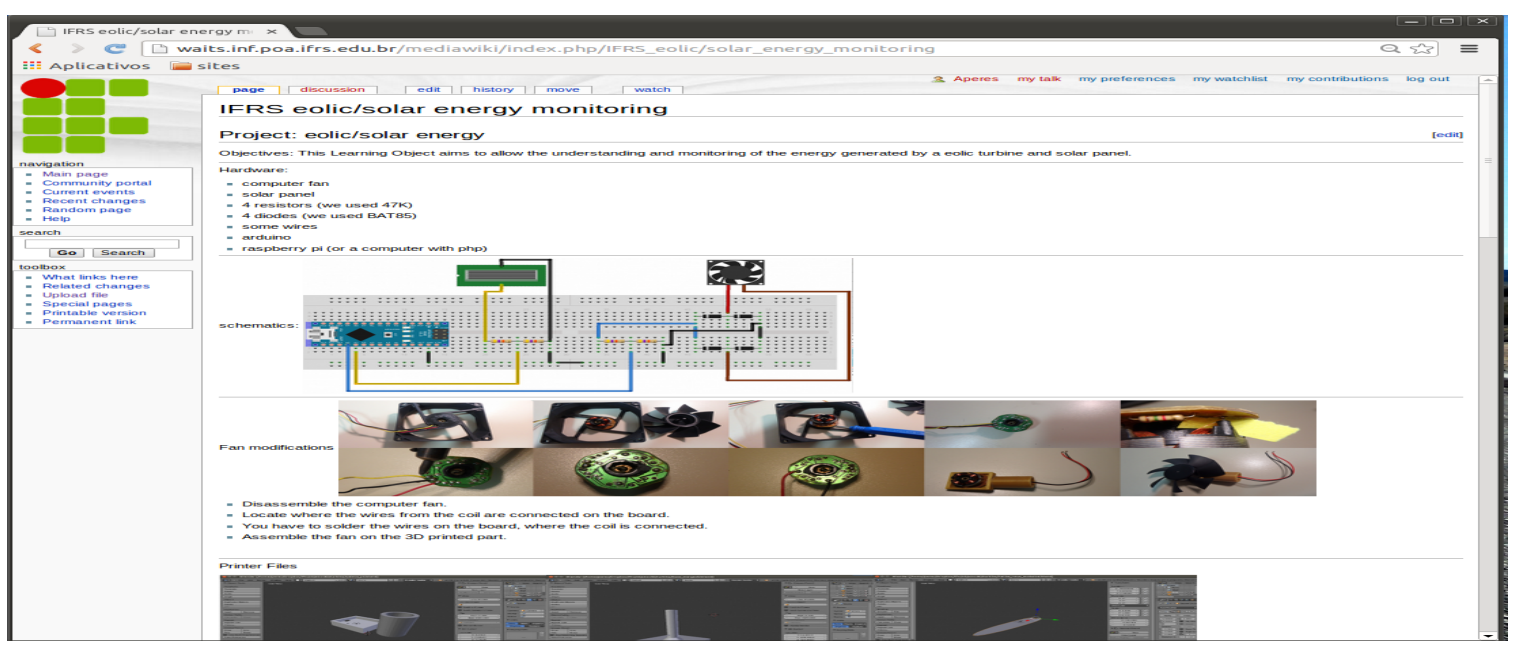

Figura 4. Página wiki

\section{Primeiros Objetos Desenvolvidos}

Até o presente momento desenvolveram-se cinco objetos. Estes objetos servem como base para atividades educacionais em diferentes áreas do conhecimento.

Os objetos desenvolvidos são descritos nos próximos subitens.

\subsection{Sensor de Ondas Eletromagnéticas}

Objetivando servir como elemento auxiliar em atividades educacionais da área de física, criou-se um objeto capaz de detectar a intensidade de ondas eletromagnéticas.

O objeto é composto por um modulo sensor, o qual visa quantificar a intensidade da interferência captada por uma antena através de gráficos. O módulo sensor se utiliza de uma antena conectada a uma placa Arduino Uno [Arduino 2013] e um computador rodando uma aplicação gráfica que traduz o sinal captado em um gráfico descrevendo em tempo real a intensidade da interferência captada.

Esse sensor se mostra útil para demonstração do conceito de indução e interferência eletromagnética. A figura 5 apresenta o modelo e interface deste objeto.

\subsection{Sensor de Condutividade da Água}

Condutividade elétrica é a capacidade de um meio de transmitir elétrons através dele. Criou-se um objeto capaz de medir a condutividade elétrica de líquidos.

O módulo é composto de um Arduino ligado a um medidor e uma aplicação que mede a condutividade através de um gráfico de barras. A figura 6 apresenta o modelo e interface deste objeto.

Este objeto foi desenvolvido em conjunto com uma equipe de professores pesquisadores do curso de Tecnologia em Gestão Ambiental da instituição de ensino à qual esta pesquisa está vinculada (dados da instituição omitidos devido à revisão). 
CBIE-LACLO 2015

Anais dos Workshops do IV Congresso Brasileiro de Informática na Educação (CBIE 2015)

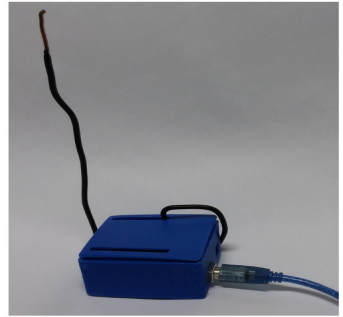

(A)

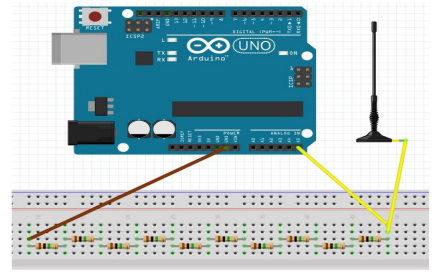

(B)

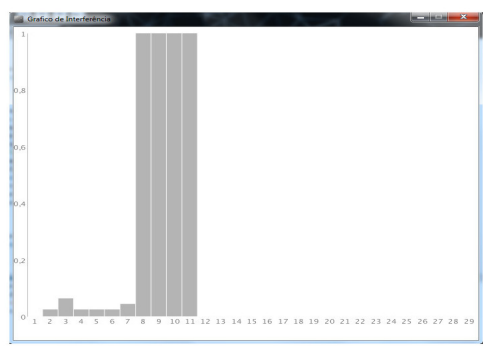

(C)

Figura 5. (A) Objeto Fabricado para Aquisição de Intensidade de Radiação Eletromagnética (B) Circuito do Objeto e (C) Interface de Apresentação dos dados
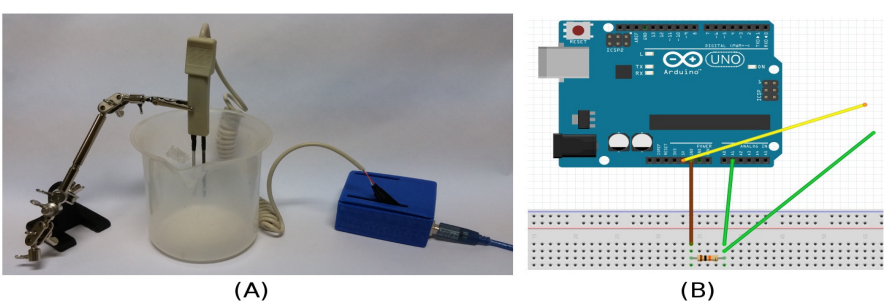

(B)

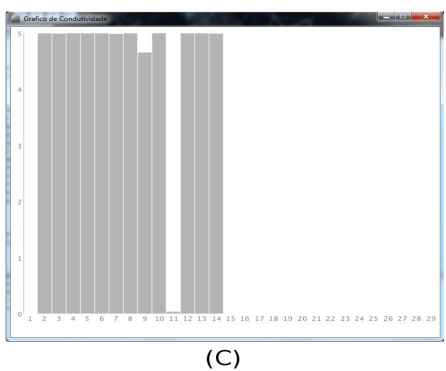

Figura 6. (A) Objeto Fabricado para Aquisição de Condutividade (B) Circuito do Objeto e (C) Interface de Apresentação dos dados

\subsection{Controle de Estufas}

É comum encontrarmos em comunidades e escolas a utilização de hortas como atividade lúdica. A utilização deste tipo de recurso permite um maior contato dos alunos com o ambiente e sociedade.

Com o objetivo de incluir novos recursos neste tipo de ambiente, criou-se um objeto capaz de realizar o monitoramento de sistemas agrícolas de pequena área.

O clima é considerado a variável mais importante na produção agrícola, pois parâmetros como luminosidade, temperatura e umidade do solo exercem influência sobre todos os estágios da cadeia de produção. A criação de estruturas de estufa auxilia no processo de controle destes parâmetros.

Pode-se listar alguns efeitos do desequilíbrio dos fatores climáticos: a falta de radiação solar pode impedir o desenvolvimento radicular da planta; temperaturas fora dos limites ideais de cada planta podem impedir ou retardar seu crescimento; pouca umidade no solo pode levar ao murchamento da planta e umidade excessiva pode impedir que o ar circule dentro do solo, possibilitando a formação de compostos tóxicos.

Foi desenvolvido um sistema computacional para monitorar e controlar os fatores mencionados, que podem impedir ou potencializar o cultivo de plantas. O sistema utiliza sensores e controladores que são gerenciados através da internet pelo usuário.

Com as informações coletadas no sistema é possível fazer um levantamento sobre cada região em que o sistema é utilizado. Com uma base de dados obtida em longo prazo é possível traçar um panorama climático e estimar as melhores espécies e variedades para a plantação em determinado região, permitindo menor intervenção humana e melhor 


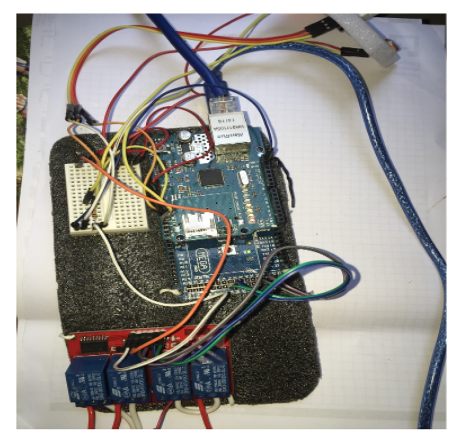

(A)

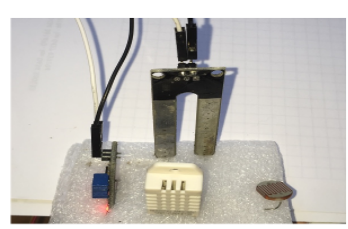

(B)

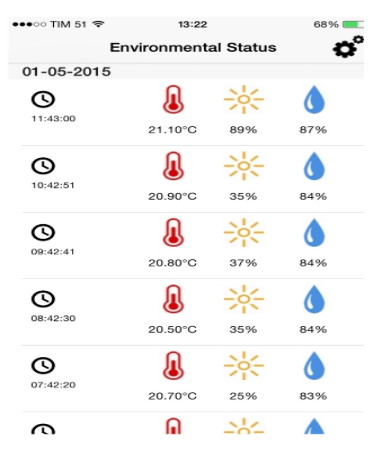

(C)

Figura 7. (A) Controlador Arduino (B) Sensores (C) Interface de Apresentação dos dados

aproveitamento do clima local. A figura 7 apresenta hardware e interface deste objeto.

\subsection{Monitoramento da Qualidade do Ar}

Criou-se um objeto capaz de obter dados sobre a qualidade do ar e disponibilizar estes dados de forma a serem utilizados em atividades de ensino.

O objeto tem como base as normativas e padrões de qualidade do ar determinadas pelo Conselho Nacional do Meio Ambiente (CONAMA). O objeto tem o intuito de determinar se o ar está dentro dos padrões e tornar pública a informação por diferentes meios de publicação.

Utilizando placas controladoras e sensores, coletam-se diferentes compostos do ar. Após a coleta, é realizada uma comparação com os padrões, a qual resultará componentes dentro ou fora dos parâmetros.

Os dados obtidos são publicados como: dados brutos (informação direta da base de dados); dados geográficos da posição da coleta (através de um mapa); e interface educacional (apresentando os efeitos fisiológicos das concentrações de compostos obtidos nos seres humanos).

Estão sendo utilizados os sensores: Sensor de Gás Metano - MQ-4, Sensor de Monóxido de Carbono - MQ-7, Sensor de Gás Hidrogênio - MQ-8, Sensor de Gás GLP MQ-6 e Sensor Óptico de Poeira.

Este objeto foi desenvolvido em conjunto com uma equipe de professores pesquisadores do curso de Tecnologia em Gestão Ambiental da instituição de ensino à qual esta pesquisa está vinculada (dados da instituição omitidos devido à revisão)

\subsection{Gerador de Energia Eólico}

Em conjunto com professores do curso de Gestão em Meio Ambiente foi realizado um levantamento dos objetos utilizados no laboratório de geração de energia existente neste curso. Este laboratório possui um conjunto de objetos que auxiliam os alunos na compreensão da geração de energia através de diferentes fontes.

O laboratório possui alguns kits comerciais para demonstração do funcionamento de baterias químicas e painéis solares. Outros objetos foram construídos pelos próprios alunos, como uma turbina eólica e outra hidroelétrica. 


\section{CBIE-LACLO 2015}

Anais dos Workshops do IV Congresso Brasileiro de Informática na Educação (CBIE 2015)

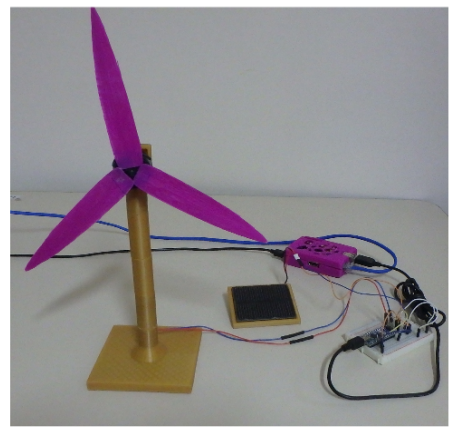

(A)

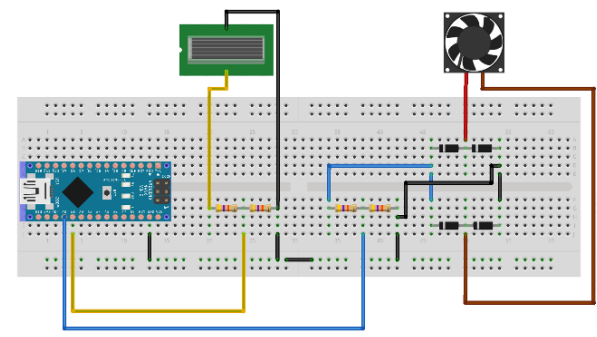

(B)

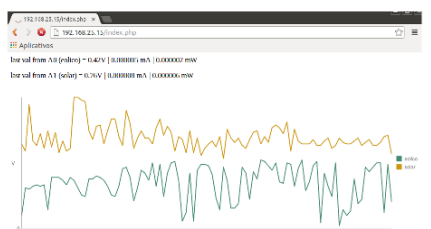

(C)

Figura 8. (A) Objeto Final (B) Circuito Utilizado (C) Interface Web

Com base neste laboratório, construiu-se um objeto capaz de gerar energia através de fonte eólica. Adicionalmente, decidiu-se por incluir neste objeto um painel solar de geração de energia.

A torre e demais peças da estrutura foram impressas utilizando-se uma impressora 3D. O objeto final contendo a turbina, torre e painel solar conectados em um Arduino Nano é apresentada na figura 8 (A). Também nesta figura nota-se a conexão do Arduino em um minicomputador Raspbery-Pi [Pi 2013], o qual foi utilizado para aquisição dos dados e apresentação dos mesmos em uma interface gráfica. A figura 8 apresenta também o circuito utilizado e interface do objeto contendo os valores de geração de energia eólica e solar.

O Raspbery-Pi possui instalado uma distribuição Linux (Raspbian) e serviços LAMP (Linux, Apache, Mysql e PHP) permitindo que os dados sejam armazenados e apresentados através de um sistema web.

\section{Conclusões}

Durante o desenvolvimento do projeto realizou-se a integração de um conjunto de soluções tecnológicas capaz de auxiliar no desenvolvimento de novos objetos a serem utilizados em atividade de ensino.

Criou-se uma infraestrutura composta por: uma rede social, responsável pela proposição, definição, coordenação e colaboração entre proponente e especialistas, servindo como base para o processo criativo da fabricação de objetos a serem utilizados em atividades educacionais; um laboratório que conta com instrumentos capazes de fabricar objetos a partir de um modelo criado por computador, e que serve como ponto de encontro presencial dos envolvidos no processo de fabricação; e um serviço de publicação do tipo wiki, também dinâmico e colaborativo, capaz de dar publicidade aos novos objetos criados por esse grupo de pessoas. Considera-se positiva a integração dos participantes do projeto na colaboração realizada nos espaços da rede social e durante o processo de fabricação e testes.

\section{Agradecimentos}

Os autores deste trabalho agradecem o apoio do CNP-q e CAPES/LIFE. 


\section{Referências}

Alves, R. M., Silva, A. L. C., Pinto, M. d. C., Sampaio, F. F., and Elia, M. d. F. (2012). Uso do hardware livre arduino em ambientes de ensino aprendizagem. In Jornada de Atualização em Informática na Educação - JAIE 2012, volume 1.

Arduino (2013). Arduino home page. http://www.arduino.cc.

Blikstein, P. (2013). Digital fabrication and 'making' in education: The democratization of invention. https://tltl.stanford.edu/sites/default/files/files/documents/publications/ Blikstein-2013-Making_The_Democratization_of_Invention.pdf.

Eychenne, F. and Neves, H. (2013). FAB LAB a Vanguarda da Nova Revolução Industrial. FabLab Brasil.

Farias, E. M. B., Netto, P. F. L., Pilletti, C. P., and Alves, E. C. M. a. (2014). A importância dos programas de extensão no ensino e prática de programação e desenvolvimento de protótipos. In Anais do XXV Simpósio Brasileiro de Informática na Educação, volume 1.

HumnHub (2015). Hum hub the flexible open source social network kit home page. https://www.humhub.org/en/overview.

Makezine (2015). Is it a hackerspace, makerspace, techshop, or fablab? http://makezine.com/2013/05/22/the-difference-between-hackerspaces-makerspacestechshops-and-fablabs/.

Martinez, S. L. and Stager, G. (2013). Invent To Learn: Making, Tinkering, and Engineering in the Classroom. Constructing Modern Knowledge Press.

MediaWiki (2014). Mediawiki home page. https://www.mediawiki.org/wiki/MediaWiki.

Mota, M. d. L. P., Campello, S. R. B. B., and Oliveira, R. R. S. (2014). Atividade e interação na rede social educacional redu. In Anais do XXV Simpósio Brasileiro de Informática na Educação, volume 1.

Oliveira, E. D. S. and Ferreira, V. V. (2014). Gênero digital wiki e a produção coletiva de texto. In Anais do XXV Simpósio Brasileiro de Informática na Educação, volume 1.

Papert, S. and Solomon, C. (1971). Twenty Things to do with a computer - Artificial Intelligence Memo 248. Massachusetts Institute of Technology.

Pi, R. (2013). Raspberry pi home page. http://www.raspberrypi.org/.

Piaget, J. (1976). To Understand IsTo Invent: The Future of Education. Penguim Books. 